\title{
ENFOQUES ANALÍTICOS EN TORNO AL COMERCIO SEXUAL DE LAS MUJERES: COORDENADAS CONTEMPORÁNEAS E INDAGACIONES EN PERSPECTIVA HISTÓRICA
}

\section{ANALYTICS APPROACHES AROUND SEX COMMERCE OF WOMEN: CONTEMPORARY COORDINATES AND INQUIRES IN A HISTORICAL PERSPECTIVE}

\section{Lucía Inés Coppa*}

RESUMEN

En este artículo se busca recomponer algunas perspectivas y enfoques presentes en las investigaciones contemporáneas en torno al mercado del sexo. Para ello, se proponen dos ejes a modo de organizar esta revisión: por un lado, se presenta una serie de claves y coordenadas contemporáneas para una aproximación a los abordajes actuales ligados a la configuración contemporánea de los mercados sexuales; por otro lado, se remiten perspectivas que historiográficamente han contribuido a conocer las tramas del comercio sexual de modo situado. La revisión conjunta de los problemas que estas indagaciones plantean, que en general suelen abordarse de manera disociada de acuerdo a su correspondencia disciplinar con estudios contemporáneos o en perspectiva histórica, puede contribuir a un diálogo fructífero habilitando terrenos de indagación a partir de nuevos enfoques o preguntas, así como, la formulación de nuevas áreas problemáticas.

PALABRAS CLAVE: MUJER * PROSTITUCIÓN * MERCADO * DESIGUALDAD * EXPLOTACIÓN SEXUAL

\section{ABSTRACT}

The aim of this article is to recompose some perspectives and focus that guide contemporary research around sex market. In that sense, I propose two axes in order to organizate the presentation. On the one hand, I present some contemporary keys and coordinates as a current approach linked to the contemporary configuration of sex markets. On the other hand, I refer to perspectives that have contributed to know the plots of sex commerce in a located way. The review of the problems that these inquiries raise, which in general are usually dealt with in a dissociated way according to their disciplinary 
correspondence with contemporary studies or in historical perspective, can contribute to an important dialogue enabling areas of inquiry based on new approaches or questions, as well as, the formulation of new problematic areas.

KEYWORDS: WOMEN * PROSTITUTION * MARKET * INEQUALITY * SEXUAL EXPLOITATION

\section{INTRODUCCIÓN}

Las investigaciones sobre el comercio sexual han ido configurando un campo en el que confluyen diversos abordajes que complejizan su espesor, en tanto espacio de significaciones a través del cual se han construido representaciones y modos de aprehender el género y las sexualidades de modo singular y situado. De esta manera es que sus estudios han girado en torno a la prostitución "en tanto práctica sexual específicamente regulada $y$ a la vez anclaje de una regulación más amplia sobre las normas que configuran la sexualidad" (Sabsay, 2011, p.110). En ese sentido, el estudio de los mecanismos histórico-sociales a través de los cuales los dispositivos sexo-genéricos son producidos, se abordan específicamente en el plano de los mercados sexuales (Rubin, 1986).

Sin pretensiones de exhaustividad, en este artículo se busca recomponer algunas perspectivas $y$ enfoques presentes en las investigaciones contemporáneas en torno al mercado del sexo, fundamentalmente en Argentina, pero retomando algunas claves analíticas transnacionales. Para ello se proponen dos ejes a modo de organizar la exposición: por un lado, se presenta una serie de claves y coordenadas contemporáneas para una aproximación a los abordajes actuales ligados a la configuración de los mercados sexuales en la actualidad; por otro lado, se remite a perspectivas que historiográficamente han contribuido a conocer distintas experiencias de comercio sexual y sus formas de control y gobierno de modo situado, así como aproximarnos a las redes $y$ vinculaciones de quienes se han desenvuelvo en sus tramas. También, se abarca aquellos campos de estudio que, sin referir a indagaciones específicas en torno al mercado del sexo, suponen referencias laterales que coadyuvan a un entendimiento más acabado de las retóricas que atraviesan su configuración.

A partir de esta revisión, se investiga transversalmente las posibilidades metodológicas y las limitaciones que estas perspectivas suponen, así como, sus contribuciones específicas para la formulación de nuevos problemas y la construcción de objetos de estudio. En particular, se focaliza en recuperar estas valiosas contribuciones para rearticular sus aportes en el horizonte del campo de estudio de la ley y la justicia en relación al mercado sexual ${ }^{1}$.

\section{TRAFFICKING, EXPLOTACIÓN SEXUAL Y TRABAJO SEXUAL AUTÓNOMO}

La prostitución ha ido ganando progresivamente un lugar importante en las agendas políticas y de investigación, así como, en los medios de comunicación. En particular, se ha revitalizado la reflexión en torno al estatuto de la prostitución a partir de los debates transnacionales suscitados en el marco de la sanción en el año 2000 del "Protocolo para prevenir, reprimir y sancionar la trata de personas, especialmente mujeres y niños"2.

1 Esta tarea se encuentra en desarrollo en el marco de la tesis de Maestría "Comercio sexual, profilaxis social y penalidad: Entramados discursivos y racionalidades en los intersticios de la sanción de la Ley Nacional 12.331 (1917-1936)" y el proyecto doctoral "Comercio sexual, abolicionismo y culturas penales: Entramados discursivos y dinámicas judiciales en la Provincia de Buenos Aires (19221965)", ambos trabajos pertenecen a la autora de este artículo.

2 El "Protocolo para prevenir, reprimir y sancionar la trata de personas, especialmente mujeres y niños" (en adelante, Protocolo de Palermo) fue debatido en el año 2000, en el marco de la Comisión para la Prevención del delito y Justicia penal de Naciones Unidas, el cual constituye uno 
En este escenario, existieron posiciones en disputa que evidenciaban una contraposición argumental, encarnada en la forma específica de lobby, de posicionamientos diferenciales respecto del ejercicio de la prostitución, de modo que lo que se tipifica como delito de trata difiere según estos posicionamientos en torno al estatuto de la prostitución (Daich y Varela, 2014). Las propuestas legales que se erigieron en ese marco como modos de definir el delito de trata de personas configuraron, en cierta medida, como correlatos de posicionamientos más amplios que versaban fundamentalmente sobre el estatuto jurídico del consentimiento de las víctimas del delito y la necesidad o no de definir medios comisivos para la configuración efectiva del tipo delictivo.

En ese sentido, existen elaboraciones que se han orientado a situar el ejercicio de la prostitución como resultado de relaciones sexo-genéricas jerárquicas y asimétricas que reproducen un modo específico de opresión hacia las mujeres que se traduce en la comprensión de la venta de servicios sexuales como una forma de esclavitud sexual (MacKinnon, 1989; Barry, 1996; Pateman, 1999). Desde esta perspectiva, para la discusión acerca del estatuto legal del consentimiento en el marco del comercio sexual, se considera que este es irrelevante jurídicamente en tanto, partiendo desde este carácter estructural de la opresión de género que atraviesa el comercio sexual, existe siempre una coerción patriarcal que hace de este consentimiento una ficción de la cual las mujeres son "víctimas" o al menos las concibe como "mujeres en situación de prostitución".

Para estas perspectivas, la sexualidad opera como base de la desigualdad, siendo precisamente el sexo definido como la condición de subordinación de las mujeres. Si el sexo es poder, esto se traduce como el poder masculino sobre las mujeres, entendiendo la subordinación femenina en términos análogos a la

de los tres protocolos adicionales a la Convención de Naciones Unidas contra el crimen organizado transnacional, junto con el "Protocolo contra el tráfico ilícito de migrantes" y el "Protocolo contra la fabricación y el tráfico ilícito de armas de fuego". subordinación de clase (Doezema, 2000). En esta línea, la prostitución emerge como una de las expresiones más extremas de la dominación masculina. Tal como Barry (1995) expone en The Prostitution of Sexuality: The Global Exploitation of Women, su trabajo ha sido:

... estudiar y exponer el poder sexual en su forma más severa, global institucionalizada y cristalizada: la prostitución - la piedra angular de toda explotación sexual [siendo] la explotación sexual una condición política, fundante de la subordinación femenina y la base sobre la que la discriminación contra las mujeres es construida $y$ performada (p. 9) 3 .

En ese sentido, se expresa Catharine MacKinnon (1989) cuando caracteriza lo que denomina la "perspectiva de la explotación sexual" para enfocar el fenómeno de la prostitución, entendiendo que allí donde las posiciones favorables al "trabajo sexual" ven una forma de agenciamiento de las mujeres en el comercio, esta perspectiva ve la prostitución como una de las más antiguas opresiones, tan extendida como la desigualdad sexual institucionalizada, de la cual la prostitución es la piedra angular.

Según expone Carole Pateman (1999), la prostitución es una de las formas en la cual el

3 La traducción es propia. El término que Barry utiliza es "enacted" del verbo "enact" que es definido por el diccionario de Cambridge como "to put something into action, especially to make something law". Se utiliza la expresión "performada", en alusión al "giro performativo" en los estudios de género, porque da cuenta del modo en que algo es actuado/puesto en acción; en lugar de referir por ejemplo al término "representada", el cual se considera que tendría algunas limitaciones en ese sentido. La autora también utilizará esta expresión cuando refiere al sexo como base de la opresión femenina: "bodied in femaleness and enacted in sexual experience" (Barry, 1995, p.278). De modo que, nuevamente, la expresión puede dar cuenta más cabalmente de que para la autora ese poder sexual estaría "encarnado" en la feminidad y "performado" en la experiencia sexual, es decir, la sexualidad misma sería el terreno en el cual la dominación se performa y se hace carne. 
hombre tiene el "acceso asegurado" a los cuerpos de las mujeres. En ese sentido, "en la prostitución, el cuerpo de la mujer, y el acceso sexual a ese cuerpo, es el objeto del contrato" (p. 60). $\mathrm{Al}$ entender el cuerpo femenino como objeto del contrato, la autora entiende que la existencia de cuerpos en el mercado se asemeja a una forma de esclavitud. La autora hace así una lectura de la prostitución en el marco de su propuesta más amplia acerca de la ficción de las formas contractuales, en las que es el "derecho patriarcal" el que se expresa en la "libertad de contrato"4.

Por eso, para MacKinnon (2009), detrás de la prostitución existe una coerción que hace del abuso sexual un sector de la economía, de modo que en estas transacciones es el dinero el que "coerciona" el sexo, antes que la garantía del consentimiento, haciendo de la prostitución una "práctica de violación serial" (p. 274). Para esta autora, "la sexualidad de las mujeres es, socialmente, algo que otros roban, venden, compran o intercambian" (1989, p.307) , entendiendo entonces que una teoría se vuelve metodológicamente feminista en la medida en que trata la sexualidad como interpretación social del poder masculino (1989, p.227), lo cual se evidencia en su propuesta de teorización feminista sobre el Estado.

Más recientemente, Jeffreys (2011) ha referido que los Estados que legalizan la prostitución se convierten en "proxenetas y explotadores" en la industria global del sexo. Según expone la autora, su investigación se inspira en el trabajo sobre prostitución de Kathleen Barry y Andrea Dworkin, buscando ampliarlo para

4 En 1988, Pateman publica El contrato sexual, en donde sostiene la tesis de que la historia política da cuenta de la creación de una nueva sociedad civil y una nueva forma de derecho político a partir de un contrato original, sobre cuya base las relaciones sociales libres remiten a la forma contractual. Según la autora, "el contrato originario es un pacto sexual-social, pero la historia del contrato sexual ha sido reprimida" (p.9), siendo el contrato social una historia de libertad, por oposición a la historia de sujeción del contrato sexual.

MacKinnon (1989) sostiene con respecto a la sexualidad que las mujeres "nunca la poseen, y los hombres nunca la tratan, ni en la ley ni en la vida, con la solicitud con que tratan la propiedad [concluyendo que] Ser propiedad sería un progreso". abordar el tema de la industria global y la variedad de sus formas. Define su enfoque entonces como un "radical enfoque feminista" considerando la prostitución como una práctica cultural nociva originada en la subordinación de las mujeres $y$ entendiéndola como constitutiva de una forma de violencia contra la mujer (Jeffreys, 2011).

En el artículo "Ouch! Western Feminists' 'Wounded Attachment' to the 'Third World Prostitute" publicado por Jo Doezema en el año 2001, un año después de la sanción del Protocolo de Palermo, se analiza la posición de la Coalition Against Trafficking in Women en el debate que tuvo lugar en torno a la definición del delito de trata de personas $y$, en particular, de los escritos de su fundadora, Kathleen Barry. En ese sentido, Doezema observa que, de acuerdo a Barry (2005) y la catw, sus análisis se fundan en la experiencia "verdadera" de las prostitutas, lo que supondría la base empírica de la teoría según la cual la prostitución, al "reducir a la mujer a un cuerpo" es necesariamente injuriante porque, en definitiva, el sexo en la prostitución es deshumanizante (2001). Sin embargo, Doezema resalta el hecho de que, en esa construcción, los testimonios de las prostitutas asumen el estatuto de verdad absoluta, $y$ pone el foco en que solo ciertas versiones son consideradas "verdaderas", construyendo la "injuria" del sexo en la prostitución de una manera circular. $\mathrm{El}$ argumento de Doezema es que, en particular, existe en dichos posicionamientos una construcción racializada en torno a las "prostitutas del tercer mundo" y que esta construcción:

... es parte de impulso más amplio del feminismo occidental para construir un 'otro' víctima, como justificación de su propio intervencionismo, de modo que el 'cuerpo injuriado' de 'la víctima de trata del tercer mundo' en los debates feministas internacionales en torno a la trata de mujeres sirve como una poderosa metáfora para el avance de ciertos intereses feministas, que no pueden asumirse como aquellos de las mismas trabajadoras sexuales del tercer mundo $(2001)^{6}$.

\footnotetext{
$6 \quad$ Traducción propia.
} 
En el artículo "Bajo los ojos de occidente. Academia Feminista y discurso colonial", publicado por Chandra Mohanty en el año 2008, se puntualiza la necesidad de abordar dos proyectos simultáneamente en relación a los "feminismos del tercer mundo"; por un lado, la crítica interna de los feminismos hegemónicos de Occidente y por el otro, la formulación de intereses y estrategias anclados en la especificidad cultural e histórica. La autora focaliza su interés en el modo de producción de la "mujer del tercer mundo" como sujeto monolítico.

Frente a la perspectiva de lo que se ha referido como "feminismo radical" en el plano global, se encuentra en los últimos años una serie de abordajes situados y generalmente, etnográficos, que conciben el ejercicio de la prostitución como producto de una decisión que conlleva diferentes grados de relativa autonomía, considerando a las mujeres que optan por su ejercicio como "trabajadoras sexuales" y abriendo por esta vía una demanda por el reconocimiento y protección de sus derechos (Pheterson, 2000; Juliano, 2005; Kempadoo, 2007; Varela, 2012; Lamas, 2016).

En el artículo "What's wrong with Prostitution? What's right with Sex Work? Comparing Markets in Female Sexual Labor", publicado por Elizabeth Bernstein en 1999, se interpela desde el sugestivo título, el trabajo de Carole Pateman "What's wrong with prostitution?", para formular otra pregunta que se asienta en una perspectiva comparativa de los mercados sexuales femeninos: si hay algo de malo con la prostitución en los términos en que es analizado por Pateman, ¿qué es lo que está bien con el "trabajo sexual"? Para ello sugiere un desplazamiento respecto de cualquier debate que parta de una esencialización de la prostitución, proponiendo a partir de su trabajo de campo:

...suspender la creencia en cualquier significado esencial de la prostitución así como considerar la viabilidad de distintas posiciones feministas en el marco de una variedad de mercados de trabajo sexual femenino, actuales e hipotéticos (1999, p.94)

Traducción propia.
En ese mismo sentido, la antropóloga Laura Agustín (2005) sugiere que existen ciertas formas "clásicas" de abordar la prostitución que no sirven para describir la realidad de la industria del sexo en sus propios términos, de modo contextualizado. Por eso, en su investigación, expone que no va a preguntarse por sus causas estructurales, por qué existe o por su definición y condiciones de posibilidad de "elegir" o no, sino más bien propone una deconstrucción del concepto "prostitución", argumentando que:

... el uso de categorías como prostitución, turismo sexual y "tráfico", por parte de representantes de gobiernos, proyectos sociales y académicos, borra la diversidad entre las situaciones e impulsos de la gente que viaja, participa en redes que facilitan los viajes $y$ vive del comercio sexual (2005, p.107).

Esta diversidad quedaría obturada si los análisis sobre el mercado del sexo parten del presupuesto de que este configura necesariamente una forma de violencia hacia las mujeres; volviendo irrisoria, en definitiva, cualquier perspectiva de análisis que no asuma este principio. La dimensión conceptual es central en las producciones que abordan el mercado del sexo, en tanto dan cuenta no solo de modalidades enunciativas, sino de la posición de la persona investigadora y sus implicaciones epistémicas.

En ese sentido, el intercambio de bienes $y$ dinero por servicios sexuales no remite unívocamente a un intercambio comercial, carente de ambigüedades, sino más bien a una construcción discursiva que es disputada y está en movimiento, mutando a través del tiempo y el espacio (Cabezas citado en Kempadoo, 2009, p. 4).

En el año 1990, Gail Pheterson llamó la atención sobre esta cuestión en una publicación en The Journal of Sex Reaserch, intitulada "The Category 'Prostitute' in Scientific Inquiry", afirmando que la categoría de "prostituta" estaba basada más en representaciones simbólicas y legales que en un conjunto de características de una población determinada, siendo la deconstrucción de esta categoría necesaria para 
poner de manifiesto el prejuicio y para conducir investigaciones científicamente válidas (p. 397). Quince años después, Weitzer (2005) reafirmará este diagnóstico considerando, a partir del análisis de tres artículos ${ }^{8}$, que frecuentemente en los estudios sobre la industria del sexo, los cánones de la investigación científica son suspendidos y la misma investigación deliberadamente sesgada en pos de una agenda política particular, y resalta la condición de sus autoras como activas referentes de campañas ligadas a la abolición de la actividad.

Ahondando en los aspectos metodológicos, Bernstein (1999) observa en esa dirección que la teorización feminista en relación a la prostitución se ha producido en gran medida desligada de trabajo empírico, aunque también afirmará que muchos de los trabajos empíricos no se han articulado con teorías feministas que den cuenta de una lectura de los mercados en clave de géneros, pero también de religión, raza y clase en sus intersecciones respectivas.

En este plano, Juliano (2005) expone que, en esta confluencia de prejuicios de base religiosa, étnicos y condicionamientos de clase, las trabajadoras sexuales padecen la mayor estigmatización en un contexto creciente de migraciones transnacionales. De modo que, según la autora, se entiende mejor el fenómeno del trabajo sexual si se lo contextualiza y se lo considera como un punto extremo de estigmatización que opera a través de la negación a la actividad de la condición y dignidad de trabajo como un gesto de violencia simbólica ${ }^{9}$. La

8 Los artículos en cuestión son Raphael, J. y Shapiro, D. (2004). "Violence in indoor and outdoor prostitution venues", Farley, M. (2004). "Bad for the body, bad for the heart: Prostitution harms women even if legalized or decriminalized" $y$ Raymond, $\mathrm{J}$. (2004). "Prostitution on demand: Legalizing the buyers as sexual consumers". Los tres textos fueron publicados en el nro. 10 de la Revista Violence Against Women. El autor caracteriza estos artículos como inscriptos en una línea de producciones sobre la industria sexual que configuran versiones de la teoría feminista radical a las que define como "extremas" en el sentido de "absolutistas, doctrinarias y no científicas" (Weitzer, 2005).

9 Para Juliano, esta estigmatización no se restringe al ejercicio del comercio sexual, sino que opera autora pone de manifiesto, asimismo, para el contexto de la Comunidad Europea, la relación inextricable que se establece conceptualmente entre migración y delincuencia, relación que legitima y realimenta fobias sociales a través del Estado.

Sobre esta cuestión, Kapur (2005) argumenta que el mismo Estado-nación y el sujeto liberal son desafiados por el fenómeno contemporáneo de las migraciones y el cruce de fronteras, asimismo, examina iniciativas estatales en el contexto de regulación de los movimientos de fronteras, entre las que evalúa la regulación legal internacional del trafficking y el impacto de la us Anti-Trafficking Act $2000^{10}$ en los derechos de las mujeres y los cruces fronterizos.

Estas iniciativas alientan respuestas estatales proteccionistas, que restringen la movilidad de las mujeres. También refuerzan las presuposiciones de que los movimientos de las mujeres son coercionados, fundamentalmente por fines sexuales y de explotación sexual, asî como de las mujeres en sí mismas como

como modelo de control sobre la sexualidad femenina en general, argumentando que "la desvalorización no es un problema que afecte sólo a los sectores estigmatizados, porque a través de la presión que se ejerce sobre ellos, en realidad lo que se procura, es disuadir a las restantes mujeres de apartarse de la norma. Es decir que la estigmatización de diferentes colectivos de mujeres es un eficaz mecanismo para controlar a las mujeres no estigmatizadas $y$ disuadirlas de infringir los modelos vigentes" (2005, p. 82).

10 El Acta de Protección a las Víctimas de Trata y Violencia del año 2000 define como objetivo el combate del tráfico de personas, especialmente en el mercado sexual, la esclavitud y la servidumbre involuntaria, así como, la reautorización de ciertos programadas federales para prevenir la violencia contra las mujeres y para otras cuestiones (la traducción es propia). "The Victims of Trafficking and Violence Protection Act of 2000 is an Act to combat trafficking in persons, especially into the sex trade, slavery, and involuntary servitude, to reauthorize certain Federal programs to prevent violence against women, and for other purposes" en https:// www.state.gov/j/tip/laws/61124.htm 
víctimas indefensas, incapaces de decidir migrar (p. 14) ${ }^{11}$.

La cuestión de las fronteras y las migraciones, desplazamientos y cruces pueden ser revisados a partir de reposicionar entonces el lugar de la experiencia como constitutiva de una comprensión espacio-temporal diferenciada. Doreen Massey (1991) menciona al respecto que:

... además de preguntarnos sobre la etnocentricidad de la idea de compresión espacio-temporal y su aceleración actual, debemos también interrogarnos acerca de sus causas: qué es lo que determina nuestro grado de movilidad y qué influencia nuestro sentido del espacio y del lugar. La compresión espacio-temporal se refiere al movimiento y la comunicación a través del espacio, a la extensión geográfica de las relaciones sociales, y a nuestra experiencia de todo ello (p. 114).

Una de las cuestiones centrales que esta perspectiva entraña es una crítica al etnocentrismo de los proyectos universalistas y liberales, en especial aquellos que se asientan en las bases de la legislación internacional y de derechos humanos. A partir de las investigaciones sobre las fronteras y los movimientos trasfronterizos de mujeres, se busca analizar estos procesos en sus propios términos, contextualizados de acuerdo a su singularidad cultural e histórica. En ese sentido, Agustín (2008) hace hincapié en cómo la racionalidad en su sentido común hegemónico occidental es una construcción cultural y en definitiva, la evaluación de la razonabilidad de los proyectos para mejorar el gobierno del comercio sexual está mediada por esa construcción en cuanto asume pretensiones universales.

La movilidad adquiere entonces una dimensión central en las propuestas analíticas expuestas, en tanto buscan redefinir las mismas condiciones de producción de conocimiento acerca de la industria del sexo, desencializando los presupuestos que podrían

La traducción es propia. orientar los cursos de investigación. Tal es el caso de la investigación de Adriana Piscitelli (2008) en la que sigue de cerca los desplazamientos de mujeres jóvenes de estratos sociales bajos de Brasil a Italia, en el marco del "turismo sexual" internacional. Para la autora, que trabaja a partir de un enfoque antropológico, "las relaciones adquieren una particular valoración en función de la posibilidad de creación de un espacio para la agencia por medio de las fronteras" (p. 182).

Tal es la comprensión que Kamala Kempadoo expone en "The war on human traffickin in the Caribbean" en el año 2007, en el cual a través de sus investigaciones define a la zona del Caribe como foco de un pánico global ligado al tráfico de personas - con un fuerte raigambre, según la autora, en antecedentes coloniales - $y$ alude a la relación intextricable entre tráfico y prostitución como una "falsa ecuación" en la que el trabajo sexual emerge necesariamente bajo la forma de una coerción que no es representativa ni da cuenta acabadamente de los modos de agenciamiento sexual de esas mujeres.

Si bien, los abordajes de la prostitución vinculados a la óptica de lo que se ha denominado "feminismo radical" dirigen su crítica a la prostitución como manifestación de una forma opresiva y de dominación, también se hallan tanto en las perspectivas pro-sex como en las propuestas analíticas de los feminismos decoloniales, siendo una forma de construir un problema de estudio que surge de un deslinde entre lo que históricamente ha sido indagado como "prostitución" y las diversas modalidades dentro de lo que podríamos denominar el "mercado del sexo". A partir de este desplazamiento, los problemas del tráfico de personas y la explotación sexual aparecen no como correlato necesario de las relaciones sexo-comerciales, sino como un fragmento dentro de lo que la antropóloga Paola Tabet (2004), referente de la corriente feminista materialista francesa, ha conceptualizado como continuum de intercambios económico-sexuales.

Estos debates en el plano local han adquirido centralidad en el marco de la adopción de políticas y legislaciones en torno a la trata de 
personas, en particular con fines de explotación sexual en los prolegómenos de la sanción de la Ley nro. 26364 del año 2008 y su posterior reforma en el año 2011. Estos acontecimientos fueron el escenario en el cual se revitalizó el debate en torno a los medios comisivos y al estatuto jurídico del consentimiento en causas por trata de personas (Colombo y Mángano, 2010), lo que propició una serie de indagaciones acerca de las experiencias y las trayectorias de las mujeres que atravesaban estos procesos $y$ del impacto en el escenario local de las políticas antitrata, así como de los procesos de judicialización en el despliegue de modalidades heterogéneas en el mercado del sexo (Daich y Varela, 2014; Varela, 2012 y 2013).

De acuerdo con las investigaciones mencionadas en los denominados "feminismos decoloniales", se ha hecho énfasis en ubicar el problema del mercado sexual en una coyuntura internacional de combate a la trata con fines de explotación sexual ${ }^{12}$, para indagar sus modos de inserción específicos en la agenda local (Daich y Varela, 2014; Varela, 2012 y 2013).

Estas investigaciones en clave local confluyen en una serie de indagaciones recientes que, fundamentalmente desde el campo de la Antropología, han profundizado en las formas en que se articula y ejerce el control de la prostitución a través de distintos agentes, mecanismos $y$ dispositivos institucionales, así como, los modos en que operan las prácticas discursivas en torno al mercado del sexo. De ese modo, estos aportes contribuyen a la agudización de los enfoques que reconstruyen las tramas en las que aparecen "las agencias y los actores que

$12 \quad$ Se refiere en particular a la "trata de personas con fines de explotación sexual", ya que, de acuerdo con el Protocolo para prevenir, reprimir y sancionar la trata de personas, especialmente mujeres y niños, que complementa la Convención de las Naciones Unidas contra la Delincuencia Organizada Transnacional, el delito de trata supone la captación, el transporte, el traslado, la acogida o la recepción de personas con fines de explotación. Esa explotación incluye tanto la explotación de la prostitución ajena u otras formas de explotación sexual como los trabajos o servicios forzados, la esclavitud o las prácticas análogas a la esclavitud, la servidumbre o la extracción de órganos. conforman la red de relaciones en que se mueven las mujeres que ejercen la prostitución" (Daich y Sirimarco, 2015, p.10).

Por esta razón es que estos abordajes privilegian el estudio de las formas de gobierno de la prostitución, atendiendo sus dinámicas específicas y prácticas cotidianas. Siguiendo la conceptualización foucaulteana, Daich y Varela (2014) fundan esta decisión metodológica en que, el "modelo de los modelos", es decir, la definición de un país, en este caso de Argentina, como abolicionista respecto de la prostitución, no puede dar cuenta de la articulación de elementos en las prácticas institucionales que exceden las premisas de un único modelo ni de las formas singulares de criminalización emergentes.

A través de estas perspectivas, las voces de las personas que realizan sexo comercial, sus experiencias, afectos $y$ trayectorias adquieren centralidad (Morcillo, 2016 y 2017). Asimismo, a partir de los problemas emergentes en el marco de la definición del delito de trata de personas, pero también de las organizaciones y movimientos sociales de trabajadoras sexuales que pugnan por el reconocimiento de sus derechos laborales, se ha intensificado el debate iusfilosófico acerca del estatus legal de la prostitución en el contexto global y en la Argentina en particular (Daich, 2012; Morcillo y von Lutzer, 2012).

Estos debates fungen como elementos centrales de las indagaciones en perspectiva histórica, en tanto conducen a una revisión de los enfoques a partir de los cuales se construyen objetos de investigación, en particular aquellos vinculados a los discursos que se han erigido sobre el tráfico de personas con fines de explotación sexual, la prostitución y el mercado del sexo en general. En su artículo, "Apresentação: gênero no mercado do sexo", la antropóloga Adriana Piscitelli (2005) observaba acerca de las contribuciones de la historiografía en esta materia que:

... son inestimables para pensar los procesos en su singularidad, pero, al mismo tiempo, en las analogías presentes en la manera en que esos movimientos de personas han sido tratados, en el pasado 
$y$ en el presente. Al comparar los resultados de las investigaciones acerca del tráfico en el pasado y en el debate actual, es posible establecer relaciones entre la intensa preocupación por esa problemática hacia fines de siglo XIX y fines de siglo $\mathrm{xx}$, dos momentos marcados, precisamente, por la profunda inquietud en relación a las migraciones (p. 10) $)^{13}$.

Doezema también avanzaba en esa dirección argumental en su artículo "Loose Women or Lost Women? The re-emergence of the myth oh 'white slavery' in contemporary discourses of 'trafficking in women'”, publicado en el año 2000, donde establece ciertos paralelismos y articulaciones retóricas entre los discursos contemporáneos vinculados al tráfico de personas, cuya emergencia se ubica hacia la década de 1980, con las historias de la esclavitud sexual, referidas entonces como "white slavery", en su uso de descripciones sensacionalistas y lenguaje emotivo. Según la autora, la repetición de esas bases discursivas conducen al interrogante acerca de la extensión y la representatividad de los casos.

13 La traducción es propia: são inestimáveis para pensar nessa singularidade e, ao mesmo tempo, nas analogias presentes na maneira como esses movimentos de pessoas têm sido tratados, no passado e no presente. Ao comparar os resultados de pesquisas sobre a construção de narrativas acerca do tráfico no pasado e o debate atual, é possível estabelecer relações entre a intensa preocupação por essa problemática nas viradas dos séculos XIX e $\mathrm{xx}$, dois momentos marcados, precisamente, pela profunda inquietação em relação à migração. Essas relações abarcam, também, a dimensão internacional dessas preocupações e do debate suscitado e seus efeitos na promulgação de leis. Nesse sentido, é importante observar que a discussão atual sobre o mercado do sexo, marcada por um interesse quase obsessivo com o tráfico de seres humanos com fins de exploração sexual, mostra o peso político da circulação das idéias através das fronteiras.
NARRATIVAS SOBRE TRÁFICO DE MUJERES Y MERCADOS SEXUALES EN PERSPECTIVA HISTÓRICA

\section{LA HISTORIA SOCIAL Y CULTURAL EN TORNO AL COMERCIO SEXUAL}

La centralidad de la retórica en torno a la "trata de blancas" y la "esclavitud sexual" es puesta de relieve en la investigación de Judith Walkowitz, Prostitution and victorian society: Women, class and the State (1982), publicada originalmente en 1980, en la cual la autora trabaja especialmente sobre los aspectos médicos, las "Contagious Diseases Acts", las revisaciones compulsivas $-y$ en ocasiones la hospitalización forzosa- sobre las mujeres británicas con sospecha de ser prostitutas durante el siglo XIX, especialmente entre 1864 y 1883. La investigación de Walkowitz, además de estudiar exhaustivamente los aspectos médicos de este período, enfatiza en los modos de agenciamiento de esas mujeres, así como, en sus espacios de sociabilidad $y$ formas de relacionarse, reponiendo una dimensión inexplorada hasta entonces ${ }^{14}$.

Walkowitz (1982 y 1993) indaga en los procesos de resistencia a la regulación de la actividad en Gran Bretaña y las movilizaciones organizadas en función de ello en las que convergían coaliciones de reformadores morales de clase media, feministas y trabajadores radicales exigiendo la revocación de la legislación sobre enfermedades contagiosas. Además, la autora identifica el papel de las campañas abolicionistas como el acceso al espacio público para mujeres de clase media, observando como contradicciones y ambigüedades del emergente movimiento de mujeres el hecho de que:

14 En una entrevista reciente realizada por Varela y Álvarez-Uría (2006), la autora reconoce que, no obstante adoptar una perspectiva deudora de la genealogía foucaltiana, debió revisar su método interpretativo, caracterizando, por un lado, su posición como "más abierta a las posibilidades de acción de los agentes sociales" y por el otro, introduciendo una dimensión de género en su analítica. 
...su lenguaje melodramático de victimización femenina privaba a las prostitutas de cualquier factor activo de subjetividad compleja: sólo podía concebirlas como víctimas inocentes falsamente atrapadas en una vida de vicio, agentes involuntarios de su propia historia, sin pasión sexual, todavía no "muertas para la vergüenza", todavía poseedoras de "recato" femenino (1993, p.404).

Sobre esta convergencia, llamaba la atención Jacques Donzelot (2008) al resaltar los vínculos entre el feminismo promocional y la filantropía moralizadora como "una alianza decisiva" (p. 42) durante la segunda mitad del siglo XIX, en particular en la lucha contra los burdeles, la prostitución y la policía de la moralidad pública en el horizonte del restablecimiento de la vida familiar de la clase obrera ${ }^{15}$.

En el mismo año de publicación del libro de Walkowitz, se publica la investigación de Paul McHugh's, Prostitution and Victorial Social Reform, focalizada más bien en cómo se organizó a nivel nacional la cruzada británica contra la regulación estatal de la prostitución -que al igual que la investigación de Walkowitz, se centra fundamentalmente en la figura de Josephine Butler-y su impacto en el sistema político.

Para el caso de Francia, la investigación de Alain Corbin es una referencia ineludible. En Women for Hire: Prostitution and sexuality in France after 1850, publicada en 1990, el autor indaga sobre las características del sistema francés que regulaba el comercio sexual a través de un sistema de registro de burdeles y supervisiones médicas y policiales periódicas desde las primeras décadas del siglo XIX. Pero, fundamentalmente, estudia el proceso posterior a 1850, a partir de las críticas crecientes que invocaban

15 Donzelot (2008) define la filantropía como una práctica discursiva que aseguraba el desarrollo de prácticas de conservación y formación de la población "que debe ser considerada como una estrategia deliberadamente despolitizante frente a la instauración de los servicios colectivos, destinada a procurarle una posición neurálgica equidistante de la iniciativa provada y de la iniciativa estatal" (p. 59). argumentos, tanto abolicionistas como neoregulacionistas en orden a desarticular el sistema reglamentario, hasta la clausura de las casas de tolerancia en 1946.

En el ámbito local, el pionero estudio de Donna Guy (1994) sobre la prostitución en la ciudad de Buenos Aires aborda la periodización 1875-1955, aunque el abordaje central y más denso remite al período de la reglamentación de la actividad, indagando de modo concomitante las vinculaciones entre trabajo, familia, clase y ciudadanía que en las regulaciones relativas a la prostitución, hallan un elemento sintomático, una imagen en la que se interceptan aquellos conceptos con modulaciones específicas ligadas al mercado del sexo. De modo que, para la autora, la prostitución emerge como "una metáfora que condensaba los temores de la clase alta y el futuro de la nación argentina" (p. 62). Es por ello que en la presentación de su obra, Guy afirmará que "la prostitución no podría separarse de su relación con el género, la reproducción y el capitalismo" (p. 13).

En el período reglamentario también se encuentra la obra de María Luisa Múgica (2014) para la ciudad de Rosario, que profundiza la indagación sobre cómo se construyó e implementó el sistema reglamentario en clave local y el modo en que el mismo combinaba aspectos político-administrativos, sanitarios y policiales, apuntalándose en "un núcleo fuerte de enunciados de corte sanitario e higiénico, con visos moralizantes" (p. 131). Una de las contribuciones centrales de la investigación de Múgica reside en la detección de una agudización de la política de control policial a partir de 1917, desentrañando una forma específica de cesión del poder político que hasta entonces era de estricto orden municipal y otorgaba primacía al control higiénico desplegado por los médicos.

Entre las características de estas investigaciones en perspectiva histórica se destacan en común dos aspectos salientes: 1) la periodización que abarca desde fines de siglo XIX hasta las primeras décadas de siglo xx y 2) el recorte espacial se realiza en torno a centros urbanos tales como Buenos Aires y Rosario, en 
un período de gran fluctuación migratoria y un aumento vertiginoso de la densidad poblacional en las ciudades portuarias. En ese sentido, y más recientemente, Marisa Miranda (2015) estudia, a través de un enfoque comparativo, aspectos vinculados a la gestión pública de la prostitución en las ciudades de Buenos Aires y Barcelona, fundando el interés en las afinidades culturales y demográficas ligadas a estos espacios urbanos, así como, en la impronta eugénico-biotipológica compartidas en torno a las construcciones vinculadas a las enfermedades de transmisión sexual en cuya clave fueron formulados discursos y políticas en torno al mercado del sexo.

Asimismo, $y$ en gran medida asociadas a estas nuevas configuraciones urbanas, en dichas investigaciones se da cuenta de una retórica de la época que atraviesa los discursos vinculados al comercio sexual en clave de "trata de blancas" $y$ adquiere centralidad de acuerdo con las historias ligadas al tráfico de mujeres que se articulaban en una dimensión internacional. En ese contexto, Argentina se representa a través de la mirada europea como lugar de destino de mujeres captadas para el comercio sexual, con la connivencia de un gobierno que permite la "trata de blancas" (Guy, 1992).

Pablo Ben (2014) recupera analíticamente este aspecto a través del estudio de folletines locales sobre la prostitución, pero, fundamentalmente, de ficciones literarias extranjeras que daban cuenta de la magnitud y las dimensiones inusitadas que la prostitución adquiría en Buenos Aires hacia el 900. Si bien, el autor no circunscribe este fenómeno a dicha ciudad y lo compara con otros nodos urbanos semejantes, tales como Shangai y Nairobi, sostiene a través de la lectura de estas fuentes que era común que se representara a Buenos Aires como destino privilegiado de mujeres para el comercio sexual.

El autor expone las argumentaciones de Guy (1994) y los cuestionamientos que su trabajo recibe respecto de las evidencias y documentaciones disponibles para aseverar que la magnitud de la prostitución en Buenos Aires fue "exagerada" por las fuentes extranjeras (Guy, 1994). Ben referirá que otros estudios señalan que:

... la migración de mujeres que trabajaban en la prostitución era hacia los años veinte un fenómeno de escala internacional. El movimiento contra la "trata de blancas" surgió como respuesta a esa oleada migratoria. Las denuncias que este movimiento realizó no fueron el mero resultado de un pánico moral en torno a la prostitución o la sexualidad de las mujeres, y si bien las representaciones moralistas abundaban, retrataban un fenómeno real que emergió con la creciente globalización (p. 21).

La problemática en torno a esta cuestión se evidencia en Las rutas de eros. La trata de blancas en el Atlántico Sur. Argentina, Brasil y Uruguay (1880-1932), una obra de la historiadora uruguaya Yvette Trochon publicada en el año 2006, en la que la atención de la autora está focalizada específicamente en el tráfico de mujeres entre fines del siglo XIx y principios del siglo $\mathrm{xx}$, tomando como espacios geográficos, tanto a Uruguay como Argentina y Brasil. Para ello, asume una perspectiva regional, lo que le permite indagar el fenómeno de trata de blancas en el período 1880-1932, sin sujetarse a las fronteras nacionales, lo que supondría obturar las dinámicas, movimientos $y$ procesos articulados en torno a una modalidad específica de tráfico.

Trochon (2003) trabaja sobre el mismo período en Las mercenarias del amor. Prostitución y modernidad en el Uruguay contemporáne, justificando este recorte en la creciente intensidad con que se perfila a partir del año 1880 "una preocupación pública por la extensión de la prostitución y la trata de blancas" (p. 13) y la inflexión que suponen los cambios en las políticas relativas al ejercicio de la prostitución a partir de 1932 en Uruguay. En esta obra, la autora destaca el interés por el estudio de las formas de interacción de las prostitutas como seres ubicuos, privilegiando entonces la dimensión intersubjetiva. Trochon destaca metodológicamente el uso de los archivos judiciales $y$ policiales como aquellos 
que posibilitan la agudización en el acercamiento a este objetivo ${ }^{16}$. Sin embargo, también refiere a la importancia de:

... rescatar los discursos que en tanto representaciones simbólicas incidieron de modo privilegiado en la construcción del fenómeno de la prostitución en la época; establecer sus determinaciones, destacar sus aspectos más relevantes, pesquisar las formas y modos en que los mismos fueron apropiados o no por los diversos actores en juego $y$, en particular, por las propias prostitutas (p. 11).

En ese sentido, si bien esta obra se circunscribe a Uruguay, en el capítulo dedicado a las enfermedades sexuales y el peligro venéreo como claves de análisis del fenómeno de la prostitución en el período analizado, la autora opta por la perspectiva regional, entendiendo que un enfoque de este tipo permite una mejor comprensión del temor a las enfermedades venéreas y la circulación de discursos asociados (2003).

Una aproximación a este aspecto realiza Schettini (2017) abordando los intercambios entre agentes de la Liga de las Naciones y las autoridades de Buenos Aires y Río de Janeiro en el marco de la primera investigación internacional sobre trata de mujeres, indagando sobre estos viajes como modos de "reforzar internacionalmente la noción de una región, América del Sur, como centro receptor de la trata de mujeres" (p. 101). Asimismo, plantea

$16 \quad$ Los estudios sobre la ley y la justicia en América Latina, orientados por los lineamientos de la Nueva historia social de la justicia, han llevado adelante un "giro en los énfasis" que implicó, a partir de la década 1980, la centralidad que adquieren la práctica judicial y los actores en dichas tramas; así como, el "descubrimiento del expediente" como fuente historiográfica (Candioti y Palacio, 2012). En ese sentido, en un Dossier publicado recientemente, se presentan trabajos específicos, enmarcados fundamentalmente en la historia de la provincia de Buenos Aires, que muestran los avances $y$ los nuevos interrogantes que las fuentes judiciales $y$ de las instituciones de seguridad $y$ del castigo han aportado $y$ aportan a la historiografía (Barreneche, 2015). las discordancias y tensiones existentes entre las iniciativas de legislación internacional y los marcos normativos, así como, los sentidos locales de la legislación antitrata que circularon entre las autoridades políticas.

El lugar de estas autoridades políticas, además, de autoridades judiciales, así como, los sentidos asociados a las formas jurídicas vinculadas al lenocinio, adquiere centralidad en las investigaciones de Schettini (2006 y 2017), en las que analiza el fenómeno de la prostitución como un prisma, como una historia "menos de cómo se efectivizó la segregación, que de una historia de los conflictos y de las dificultades que marcaron ese proceso" (2017, p.24). En la investigación de Schettini confluyen diferentes dimensiones del comercio sexual, tanto como experiencia de trabajo y sociabilidad, así como, factor de estigma y justificativo de políticas públicas de control.

Estas investigaciones han supuesto una contribución sumamente importante a los estudios históricos sobre el mercado de sexo en clave latinoamericana, haciendo hincapié en las particularidades locales a través de las cuales cobraron sentido los marcos globales vinculados al tráfico de mujeres, pero también dando cuenta de la singularización propia de las agencias institucionales emergentes en sus intersticios.

En clave local, Grammático (2000) delinea algunos aspectos de esta dimensión al abordar la transición del modelo reglamentario al abolicionismo de los reglamentos. La autora expone que durante décadas se erigieron voces que cuestionaban el modelo reglamentario, cuyo basamento residía en la denuncia de los métodos coercitivos sobre las prostitutas y el avasallamiento a sus derechos individuales, además la ineficacia en el control de la propagación de enfermedades venéreas para lo cual la reglamentación se había configurado como herramienta estatal. Frente a estas voces, la autora plantea un interrogante que radica en preguntarse por las condiciones, situaciones $y$ hechos que habilitan el consenso hacia la materialización de las aspiraciones antirreglamentaristas hacia la década de 1930, en los prolegómenos de la sanción de la Ley 12.331 de Profilaxis Social de las Enfermedades Venéreas. 
ENFERMEDAD-EROGENEIDAD COMO "COMPLEJO INDISOLUBLE”: HISTORIA DE LA SALUD Y LA ENFERMEDAD EN CLAVE DE GÉNEROS Y SEXUALIDADES

La indagación sobre el fenómeno de la prostitución en perspectiva histórica supone recuperar las claves de inteligibilidad de época y las categorías disponibles a través de las cuales se configuraron los discursos que se erigieron en torno a este comercio. En ese sentido, es insoslayable el estudio de los modos en que la preocupación por la propagación de enfermedades venéreas, así como, los pánicos morales asociados a las sexualidades (Walkowitz, 1982), coadyuvaron a la emergencia de formas discursivas específicas, a la puesta en discurso (Foucault, 2002) de preocupaciones atinentes al mercado del sexo.

De ese modo, se dialoga con antecedentes que remiten a campos de estudios que contribuyen a la complejización del análisis propuesto. Entre fines del siglo XIX y principios del xx, la prostitución aparece como objeto de debate vinculado a la preocupación por la salud - tanto del cuerpo individual como del cuerpo social de la Nación-y ligado estrechamente a la propagación de enfermedades venéreas, de modo que el vínculo enfermedad-erogeneidad configura un complejo indisoluble (Butler, 2009) como clave de análisis de la época. Para analizar este aspecto, las producciones vinculadas a la investigación en los campos de la Historia de la Salud brindan herramientas en el abordaje de la enfermedad como construcción social $y$ en el estudio de sus significaciones culturales (Lobato, 1996; Armus, 2002). En ese sentido, Susan Sontag (2012) referirá las implicancias subjetivas y emotivas de la enfermedad y su hibridación metafórica en términos socioculturales. Según expresa "basta ver una enfermedad cualquiera como un misterio, $y$ temerla intensamente, para que se vuelva moralmente, si no literalmente, contagiosa" (p. 1).

Las enfermedades epidémicas no son una novedad en el período analizado, sin embargo, adquieren significaciones singulares de acuerdo a las nuevas características urbanas y poblacionales, así como, su correlato en las retóricas del progreso y el orden, y las políticas higienistas.
Como señala Armus (2002), si bien, las agendas de los grupos higienistas fueron mutando desde mediados de siglo xIx hasta entrado el siglo xx como reflejo de los cambios acaecidos en el mundo urbano, la ciudad como artefacto y trama de relaciones configuró un núcleo perdurable de sus preocupaciones. Las epidemias finiseculares y los pánicos emergentes en torno al contagio confluyeron en progresivos procesos de medicalización de las problemáticas asociadas a la urbanidad. Los lenguajes médicos configuraron una gramática específica a través de la cual los nuevos problemas socioculturales se volvían inteligibles en clave higiénica.

La circulación de discursos y prácticas institucionales en torno a "focos infecciosos" alcanzaron la proyección de políticas que confluyeron en las diferentes reglamentaciones locales en torno a la prostitución, dirigidas en uno de sus aspectos centrales a las revisaciones médicas periódicas de las prostitutas. María Luisa Múgica (2016) señala que la prostitución, en el marco de la propagación creciente de enfermedades venéreas, se representaba como el principal foco de difusión de estas. Del mismo modo, a través de los estudios culturales de la ciencia (Miranda y Vallejo, 2012) se remite a las configuraciones de la salud pública $-y$ su híbrida frontera con la moralidad pública en su cristalización normativa- así como, a la progresiva medicalización de la sexualidad, como modo de exposición de la inextricable vinculación entre la prostitución y la enfermedad, además, lateralmente, al cuerpo de la prostituta como "foco infeccioso".

Sin embargo, desde las preocupaciones higienistas en torno a la salud pública, se puede advertir un desplazamiento hacia la retórica que sitúa el problema de la propagación de enfermedades venéreas en el marco de un hipotético escenario de degeneración de la raza (Miranda, 2011). De modo que el factor disgénico que supone la propagación de enfermedades venéreas adquiere un estatuto central en las reformulaciones discursivas hacia la década de 1930, lo cual funge como correlato de un proceso de consolidación paradigmática e institucional del discurso eugénico en el país (Miranda, 2012; Ledesma y Valobra, 2012). En este escenario, 
Biernat (2007 y 2013) plantea la hipótesis de que si bien, hacia los años 30 se cimienta un consenso acerca de la amenaza para el acervo racial que constituye la prostitución, en tanto principal propagadora de las enfermedades venéreas, su consideración como "mal necesario" dificulta un acuerdo acerca de la instauración del sistema abolicionista.

Se entiende que las construcciones socio-médicas que configura el andamiaje discursivo en torno al mercado del sexo, se insertan asimismo en una matriz simbólica que moldea una trama emotiva, representaciones, imágenes $y$ formas de legitimación de políticas en las que los cuerpos y las sexualidades son constitutivas y revelan tramos decisivos de sus dinámicas (Acha, 2013).

\section{HISTORIA DEL CONTROL SOCIAL Y DE LAS INSTITUCIONES}

En tanto objeto de investigación histórica, además de los estudios específicos en materia de comercio sexual, los abordajes del fenómeno de la prostitución desde finales del siglo XIX comienzan a advertirse de manera tangencial y secundaria en investigaciones que focalizan sus intereses en la inteligibilidad de amplios fenómenos sociales implicados en el proceso de modernización social y consolidación del Estado argentino, con su consiguiente recepción de flujos migratorios y un crecimiento poblacional exponencial (Zimmermann, 1995; Suriano, 2004). Ello así, dando lugar a la emergencia de preocupaciones atinentes al robustecimiento del "ser nacional" en ciernes y concomitantemente, al debate sobre la formación de la agenda pública y las estrategias políticas en ese entramado, en el que el fenómeno de la prostitución emerge y es leído como elemento sintomático.

Asimismo, se encuentran referencias laterales en estudios ligados a la investigación en el campo de la historia del control social, tanto desde el análisis de estrategias tendientes a la "defensa social" como de los dispositivos institucionales que configuraron el entramado "técnico" del poder a través de una historia de las instituciones (Di Liscia y Bohoslavsky, 2005). Es en esa línea que se inscriben trabajos que específicamente ahondan en las relaciones entre policía y prostitución a través de los registros fotográficos y sus efectos de subjetividad.

Di Liscia, Billorou y Rodríguez (1999) realizan una caracterización de la prostitución en el territorio nacional de La Pampa a partir de las dimensiones del registro y las fotografías policiales, asimismo, atisbando posibles estrategias que las mujeres emplearon para sortear el estigma social que el oficio implicaba. Por otro lado, Daich y Sirimarco (2012) retoman este trabajo caracterizando la relación entre policía y prostitución como pornográfica, definiendo lo pornográfico como "el ejercicio de producción de grupos inaccesibles a la mirada, de grupos cuya visión debe ser objeto de custodia estatal" (p. 84). Las autoras indagan la Serie "Registro de Prostitutas" del Fondo "Jefatura de Policía" del Archivo Histórico Provincial de La Pampa para reflexionar, conforme ellas mismas aclaran, en torno a la relación policía-prostitución en términos generales, en su carácter representativo más allá de las especificidades y particularidades locales.

Otro aspecto que coadyuva en la indagación sobre el mercado del sexo son las reelaboraciones contemporáneas de perspectivas sociológicas y antropológicas imperantes en el campo de la criminología para el período propuesto (Sozzo, 2009; Anitua, 2010; Salvatore, 2010) en las que la figura de la prostituta remite a la configuración femenina del sujeto "peligroso" o "desviado", mediado por las categorías de anomalía y perversión, con una fuerte impronta del positivismo criminológico (Dalla-Corte, 1996; Cesano y Dovio, 2009).

Cesano y Dovio (2009) revisan la cuestión de la criminalidad femenina en el marco de dos publicaciones científicas: los Archivos de Criminología, Psiquiatría y Ciencias Afines y la Revista de Criminología, Psiquiatría y Medicina Legal, indagando sobre el grado de representación de la mujer en los discursos expertos de la época, en particular, en el discurso científico del positivismo criminológico. En la exhumación de estos archivos, los autores dedican un apartado a la prostitución femenina en el marco de la distinción efectuada entre el delito 
y la mala vida, como concepto en el que recaían conductas y comportamientos antisociales en la lucha por la vida y que excedían la noción y definiciones penales.

\section{CONCLUSIONES}

Las investigaciones en torno a los mercados sexuales, tanto contemporáneos como en perspectiva histórica, confluyen en un campo de estudios que se encuentra en un proceso de acrecentamiento, proceso que tiene su correlato en las discusiones políticas y jurídicas vigentes acerca de su configuración. Este artículo pretendió bosquejar una serie de coordenadas que den cuenta de algunas de las perspectivas $y$ enfoques que las orientan, sin ahondar en las particularidades $y$ las dimensiones singulares de cada una de las investigaciones referenciadas.

Para ello, se desglosó esta presentación en dos ejes centrales. En primer lugar, se presentó a través de una serie de investigaciones recientes que abordan los mercados sexuales en clave contemporánea, algunos de los ejes que las guían y de los argumentos que emergen en ese marco. En segundo lugar, se revisaron indagaciones en perspectiva histórica que han contribuido de manera central a conocer el mundo de la prostitución desde fines del siglo XIx. Además, se intentó pensar de qué modo, investigaciones que se corresponden con campos de estudios más amplios, aún no tematizando la prostitución en su singularidad, confluyen en la indagación de las tramas significantes en torno al comercio sexual, tal como los desarrollos desde la Historia de la Salud y la Enfermedad y la Historia del control social o de las Instituciones.

Se considera que la revisión conjunta de los problemas que estas indagaciones plantean, que en general suelen abordarse de manera disociada de acuerdo a su correspondencia disciplinar con estudios contemporáneos o en perspectiva histórica, pueden contribuir a un diálogo fructífero. Sin perjuicio de la singularidad y el carácter situado que ellas suponen, su lectura común y recíproca contribuye a visualizar áreas de indagación y matrices conceptuales que pueden eventualmente ser revisitados a partir de nuevos enfoques o preguntas, así como, a la formulación de nuevas áreas problemáticas.

\section{REFERENCIAS}

Agustín, L. (2005). La industria del sexo, los migrantes $y$ la familia europea en Guasch, O. y Viñuales, O. (coords.). Sexualidades: Diversidad y Control Social. Barcelona: Editorial Bellaterra.

Agustín, L. (2008). Sex and The Limits of Enlightenment: The Irrationality of Legal Regimes to Control Prostitution. Sexuality Research \& Social Policy, 5 (4).

Anitua, I. (2010). Historias de los pensamientos criminológicos. Bs. As: Editores del Puerto.

Armus, D. (2002). La enfermedad en la historiografía de América Latina moderna. Asclepio LIV(2).

Barry, K. (1995). The prostitution of sexuality. Nueva York: NYU Press.

Barry, K. (1996) Prostitution, sexual violence, and victimization: Feminist perspectives on women's human rights en Viano, E. (ed.). Victim's Righsts and Legal reforms: International perspectives. Onait.

Ben, P. (2014). Historia global y prostitución porteña: el fenómeno de la prostitución moderna en Buenos Aires, 1880 1930. Revista de Estudios Maritimos y Sociales, 5/6.

Bernstein, E. (1999). What's Wrong with Prostitution? What's Right with Sex Work? Comparing Markets in Female Sexual Labor. Hastings Women's Law Journal 10.

Biernat, C. (2007). Médicos, especialistas, políticos y funcionarios en la organización centralizada de la profilaxis de las enfermedades venéreas en la Argentina (19301954). Anuario de Estudios Americanos, 64(1), 257-288.

Biernat, C. (2013). Entre el abolicionismo y la reglamentación: prostitución y salud pública en Argentina (1930-1955) (19301955). Cuadernos del Sur, 40.

Candioti, M. y Palacio, J.M. (comp.) (2012). Justicia, derecho y política en América Latina. Buenos Aires: Prometeo. 
Colombo, M. y Mángano, A. (2010). El consentimiento de la víctima en la trata de personas y un análisis sobre los medios comisivos previstos en el Código Penal. Revista del Ministerio Público, 11. Buenos Aires, Argentina.

Corbin, A. (1990). Women for Hire: Prostitution and sexuality in France after 1850. Cambridge, Massachusetts: Harvard University Press.

Daich, D. (2012) ¿Abolicionismo o reglamentarismo? Aportes de la antropología feminista para el debate local sobre la prostitución. Revista RUNA, XXXIII (1), 71-84.

Daich, D., y Sirimarco, M. (2012). Policía y prostitución. Una relación pornográfica (El control de la prostitución en Argentina 1875-1936). Manizales 9(1), 80-100.

Daich, D., y Sirimarco, M. (2014). Policía y prostitución. Una relación pornográfica (El control de la prostitución en Argentina 1875-1936). Publicar, XVII.

Daich, D., y Sirimarco, M. (coord.). (2015). Género y violencia en el mercado del sexo. Política, policía y prostitución. Buenos Aires: Editorial Biblos.

Daich, D., y Varela, C. (2014). Entre el combate a la trata y la criminalización del trabajo sexual: las formas de gobierno de la prostitución. Revista Delito y Sociedad 38.

Dalla-Corte Caballero, G. (1996). Discusión sobre la influencia de la corriente criminológica positivista en el discurso penal argentino. Revista Gimbernat, 26, 157-169.

Di Liscia, M.H., Billorou, M.J., y Rodríguez, A.M. (1999). Prostitutas: Registros y fotos. En D. Villar, María de L. Historia y género: seis estudios sobre la condición femenina. Buenos Aires: Biblos.

Di Liscia, M. S., y Bohoslavsky, E. (editores) (2005). Instituciones y formas de control social en América Latina, 1840-1940. Buenos Aires: Prometeo.

Doezema, J. (2000). Loose Women or Lost Women? The re-emergence of the myth oh 'white slavery' in contemporary discourses of 'trafficking in women'. Gender Issues 18 (1), 23-50.
Doezema, J. (2001). 'Ouch! Western Feminists' 'Wounded Attachment' to the 'Third World Prostitute'. Feminist Review 67, 16-38.

Grammático, K. (2000). 'Obreras, prostitutas y mal venéreo. Un Estado en busca de la profilaxis'. En F. Gil, V. Pita y M.G. Ini (dir). Historia de las mujeres en la Argentina SXX. Buenos Aires: Taurus.

Guy, D. (1992). White Slavery, Citizenship and Nationality in Argentina. White Slavery and Mothers Alive and Dead: The Troubled Meeting of Sex, Gender, Public Health and Progress in Latin America. Lincoln: University of Nebraska Press.

Guy, D. (1994). El sexo peligroso. La prostitución legal en Buenos Aires 1875-1955. Buenos Aires: Sudamericana.

Jeffreys, S. (2011). La industria de la vagina. La economía política de la comercialización global del sexo. Buenos Aires: Paidós.

Juliano, D. (2005). El trabajo sexual en la mira. Polémicas y estereotipos. Cadernos pagu 25, 79-106.

Kapur, R. (2005). Erotic Justice: Law and the New Politics of Postcolonialism. London: GlassHouse.

Kempadoo, K. (1999). Sun, Sex, and Gold: Tourism and Sex Work in the Caribbean. Rowman \& Littlefield.

Kempadoo, K. (2007). The war on human trafficking in the Caribbean. Race and Class 49 (2), 79- 84.

Kempadoo, K. (2009). Caribbean sexuality: mapping the field. Caribean Reviw of Gender Studies. Issue 3.

Lamas, M. (2016). El fulgor de la noche. El comercio sexual en las calles de la Ciudad de México. México: Océano.

Ledesma, N., y Valobra, A. (2012). Eugenesia y derechos: idearios médico-políticos sobre los roles públicos y privados de las mujeres, 1930-1945. En M. Miranda y G. Vallejos (dir.). Una historia de la eugenesia. Argentina y las redes biopoliticas internacionales, Tomo I: 1912-1945. Buenos Aires: Editorial Biblos.

Lobato, M. Z. (ed.) (1996). Política, médicos y enfermedades. Lecturas de historia de la 
salud y la enfermedad en la Argentina. Buenos Aires: Biblos UNMDP.

MacKinnon, C. (1989). Hacia una teoría feminista del Estado. Universitat de Valencia, Ediciones Cátedra.

MacKinnon, C. (2009). Trafficking, Prostitution and Inequality. Harvard Civil RightsCivil Liberties Law Review 46.

Massey, D. (1991). Un sentido global del lugar. A. Albet y N. Benach (trad.). Marxism Today, 24-29.

Miranda, M. (2011a). Buenos Aires, entre Eros y Tánatos. La prostitución como amenaza disgénica (1930-1955). Dynamis 32 (1), 93-113.

Miranda, M. (2011b). Controlar lo incontrolable. Una historia de la sexualidad en la Argentina. Buenos Aires: Biblos.

Miranda, M. (2015). 'Sobre las "asalariadas del amor": prostitución y norma (Argentina y España, fin-du-siècle). Revista Trabajos y Comunicaciones, 42.

Miranda, M. y Vallejo, G. (2012). Una historia de la eugenesia: Argentina y las redes biopolíticas internacionales. Buenos Aires: Biblos.

Mohanty, C. (2008). Bajo los ojos de Occidente. Academia feminista y discurso colonial. En L. Suárez y A. Hernández (ed.). Descolonizando el Feminismo: Teorías y Prácticas desde los Márgenes. Madrid: Ed. Cátedra.

Morcillo, S. (2016). El debate feminista sobre la prostitución. ¿Una polarización poco fecunda? Villa María: Universidad Nacional de Villa María.

Morcillo, S. (2017). Contrabando de afectos, fugas de capitales y otros desplazamientos. Relaciones de sexo comercial más allá de las fronteras de la prostitución. Cadernos pagu, 49.

Morcillo, S. y Von Lurzer, C. (2012). 'Mujeres públicas' y sexo clandestino. Ambigüedades en la normativa legal sobre prostitución en la Argentina'. En D. Jones, C. Figari y S. Barrón López (coord.). La producción de la sexualidad. Politicas y regulaciones sexuales en Argentina. Buenos Aires: Biblos.
Múgica, M. L. (2001). Sexo bajo control: la prostitución reglamentada: un escabroso asunto de política municipal. Rosario entre 1900 y 1912. Rosario: UNR Editora.

Múgica, M. L. (2014). La ciudad de las Venus impúdicas. Rosario, historia y prostitución, 1874-1932. Rosario: Laborde Libros Editor.

Múgica, M. L. (2016). 'Males vergonzantes' y prostitución reglamentada. Rosario, Argentina (1874-1932). Asclepio. Revista de Historia de la Medicina y de la Ciencia, 68 (2).

Pateman, C. (1999). What's wrong with prostitution? Women's Studies Quarterly, 1/2 (27), 53-64.

Pheterson, G. (1990). The category 'Prostitute' in Scientific Inquiry. The Journal of Sex Research, 3 (27), 397-407.

Pheterson, G. (2000). 'El prisma de la prostitución'. Madrid: Talasa.

Piscitelli, A. (2005). Apresentação: gênero no mercado do sexo. Cadernospagu, 25, 7-23.

Piscitelli, A. (2008). Industria del sexo y mercado matrimonial: La migración brasileña hacia Italia en el marco del -turismo sexual- internacional. En G. Herrera, J. Ramírez (eds.). América Latina migrante: estado, familias, identidades. Quito, Ecuador: Flacso, Ministerio de Cultura del Ecuador.

Rubin, G. (1986). El tráfico de mujeres: Notas sobre la 'economía política' del sexo en México DF. Revista Nueva Antropología, 30 (VIII).

Sabsay, L. (2011). Fronteras Sexuales. Espacio urbano, cuerpos y ciudadanía. Buenos Aires: Paidós.

Schettini, C. (2006). Que tenhas teu corpo: uma história social da prostituição no Rio de Janeiro das primeiras décadas republicanas. Rio de Janeiro: Arquivo Nacional.

Schettini, C. (2017). En búsqueda de América del Sur: agentes secretos, policías y proxenetas en la Liga de las Naciones en la década de 1920. Revista Iberoamericana, 64 (xvII), 81-103. 
Sontag, S. (2012). La enfermedad y sus metáforas/El sida y sus metáforas. Buenos Aires, Argentina: Editorial Debolsillo.

Tabet, P. (2004). La grande arnaque. Sexualité des femmes et échange économicosexuel. Paris: L'Harmattan, Bibliothèque du Féminisme.

Trochon, Y. (2003). Las mercenarias del amor. Prostitución y modernidad en el Uruguay (1880-1932). Montevideo: Taurus.

Trochon, Y. (2006). Las rutas de Eros. La trata de blancas en el Atlántico Sur. Argentina, Brasil y Uruguay (1880-1932). Montevideo: Taurus.

Varela, C. (2012). Del tráfico de las mujeres al tráfico de las políticas. Apuntes para una historia del movimiento anti-trata en la Argentina (1998-2008). Revista Publicar, XII, 35-64.

Varela, C. (2013). De la "letra de la ley" a la labor interpretante: la "vulnerabilidad" femenina en los procesos de judicializacion de la ley de trata de personas (20082011). Cadernos pagu, 265-302.

Varela, C. (2015). La campaña antitrata en Argentina y la agenda supranacional. En
D. Daich y M. Sirimarco (coord.). Género $y$ violencia en el mercado del sexo. Política, policía y prostitución. Buenos Aires: Biblos.

Varela, J., y Álvarez-Uría, F. (2006). La voz de los archivos: Mujeres, conflictos urbanos y sexualidad. Entrevista a Judith Walkowitz. Alcalá, Minerva. Revista del Círculo de Bellas Artes, 3.

Walkowitz, J. (1982). Prostitution and victorian society: Women, class and the State. Cambridge University Press.

Walkowitz, J. (1993). Sexualidades peligrosas. En Duby, G. y Perrot, M. Historia de las mujeres en Occidente: el siglo XIX: cuerpo, trabajo y modernidad: actividades y reivindicaciones, tomo 8. Madrid: Taurus.

Weitzer, R. (2005). New direction in research of prostitution. Crime, Law and Social Change, 43.

Fecha de ingreso: 07/07/2018 Fecha de aprobación: 10/10/2018 